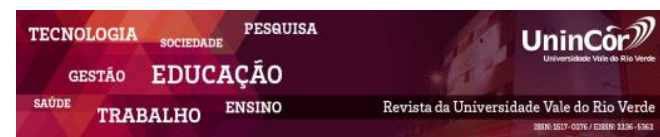

Revista da Universidade Vale do Rio Verde ISSN: 1517-0276 / EISSN: 2236-5362 v. 17 | n. 1 | Ano 2019

Elia Machado de Oliveira Universidade Estácio de Sá - ESTÁCIO elia561@hotmail.com

Wellington Fernando da Silva Ferreira Centro Universitário Campos de Andrade UNIANDRADE wellingtonferreira42@gmail.com

\section{DILEMAS NO PROCESSO DE AUDITORIA: PARADOXOS DO SERVIÇOS EM SAÚDE}

\begin{abstract}
RESUMO
A presente revisão objetiva compreender a dinâmica do processo da auditoria e suas relações com a gestão hospitalar. Metodologicamente optou-se por um estudo descritivo exploratório qualitativa através de uma revisão sistemática de literatura, realizou-se a busca de estudos em periódicos nacionais referente aos últimos cinco anos, e amostra final constituída por 16 textos científicos completos. Nota-se a relevância da auditoria no atual cenário das instituições de saúde, ajudando nas esferas de custos e qualidade dos serviços prestados, a utilização da auditoria como uma ferramenta de gestão deve observar as dinâmicas das ações e serviços de saúde, e intensificar a capacitação e treinamento permanente de todos os envolvidos e uma gestão da qualidade contribui serviços prestados e organizações com o proposito final de atender com eficiência os usuários. Por fim, aspectos de interconexões acerca da explicações e extensões do instrumento de auditoria no campo da saúde na contemporaneidade foram obtidos, e suas implicações, por meio desta averiguação proporcionam proeminência social, profissional e acadêmica, mesmo com relatos do crescimento do alento a problemática percebeu-se que há escassez da literatura sobre a luz da temática desta forma, evidencia-se a necessidade da realização de novos estudos a fim de investigar e analisar, os processos de auditoria.
\end{abstract}

Palavras-chave: Auditoria em Saúde; Enfermagem; Gestão de Qualidade.

\section{AUDIT PROCESS DILEMMAS AND ITS PARADOX IN HEALTH SERVICES}


contemporaneous ones, in the same way as in the past, action sociale, professionnelle et académique, plus aussi dans le cadre d'une problématique. it is evident that there is a shortage of the literature on the light of the theme in this way, it is evident the need to carry out new studies in order to investigate and analyze the audit processes.

Keywords: Health Audit; Quality Management; Nursing.

Recebido em: 31/07/2018 - Aprovado em: 26/03/2019 - Disponibilizado em: 15/07/2019

\section{INTRODUÇÂO}

A gênese da denominada auditoria surge da indulgência da necessidade de confirmação de investidores e proprietários, acerca de seus andamentos de realidade econômico financeira referente a patrimônio das empresas/industrias investidas, em virtude do advento de grandes empreendimentos e simultâneo ao desenvolvimento da economia da época, que propiciou participação e formação do capital (PASSOS, et al. 2012; ALELUIA e SANTOS, 2013; SIQUEIRA, 2014; OLIVEIRA, et al. 2017; GARCIA, et al. 2018).

Conceitos e definições de auditoria estigmatizam a um sistema de revisão e controle, para informar a administração e/ou gestão acerca da eficiência e eficácia dos programas em incremento no âmbito da saúde e empresarial pois a função não é exclusivamente indicar as falhas e problemas, mas apontar sugestões (D'INNOCENZO, et al. 2010; SILVAI, et al. 2012; MOIMAZ, et al. 2012; SILVA e SANTO, 2013; FILHO, et al.2016).

Neste contexto, a auditoria em saúde na contemporaneidade cresce seu alento no cotidiano, haja vista o aumento da demanda a exigências do mercado de trabalho, quais tornam-se necessária aos profissionais capacitados para operacionalizar a dinâmica de auditoria, proporcionando a qualidade do serviço, ajustando falhas, para um resultado satisfatório (SCARPARO, et al. 2008; ROSA, et al. 2012; CLAUDINO, et al. 2013; SILVA e SANTO, 2013; CARMO, 2017).

Deste modo, com o acréscimo da demanda no mercado de trabalho na área da auditoria em saúde, qual se tratar de uma atividade recente a âmbito nacional, quais revelam-se poucos estudos abordando a importância desta temática, surge a necessidade de abarcar a necessidade da auditoria em saúde para observar a qualidade da assistência (CAMELO, et al. 2009; SANTOS, et al. 2013; CECCON, et al. 2013; SIQUEIRA, 2014; COSTA, 2017).

Em linhas gerais, custos hospitalares expõem indicadores de grande proporção, existem duas modalidades de auditoria: a interna e a externa, com finalidade de aferir a preservação dos padrões instituídos e proceder ao arrolamento de dados que permitam avaliar a qualidade, custos e os gastos na saúde (D'INNOCENZO, et al. 2010; SILVAI, et al. 2012; MOIMAZ, et al. 2012; SILVA e SANTO, 2013; GARCIA, et al. 2018). 
Portanto, a auditoria interna concretizada na instituição pela equipe da instituição ou nas instalações das operadoras de planos de saúde, a auditoria externa que acontece na instituição (hospitais e clínicas) pelo SUS e prestadora, a auditoria externa é realizada por um profissional não pertencente a empresa, auditor externo tem como desempenho a verificação da adequação patrimonial e financeira das demonstrações contábeis (SCARPARO, et al. 2008; ROSA, et al. 2012; CLAUDINO, et al. 2013; SILVA e SANTO, 2013; CARMO, 2017).

Contudo, auditoria interna quanto a externa são de grande relevância para a empresa hospitalar, a atividade de auditoria hospitalar tem se destacado como ferramenta de gestão e fiscalização mais apropriado às precisões de gerenciamento das informações no ambiente hospitalar, servindo como instrumento de gestão, pois por meio de um método longo e minucioso constata anormalidades no prontuário médico, como também na área contábil e qualidade de serviços (CAMELO, et al. 2009; SANTOS, et al. 2013; CECCON, et al. 2013; SIQUEIRA, 2014; COSTA, 2017).

Diante do exposto, justifica-se a relevância supracitada da temática, deste modo esta revisão pretende como objetivo; compreende a dinâmica do processo da auditoria nas relações com a gestão hospitalar, para tal, respondendo a seguinte questão: quais perspectivas as dimensões, e dilemas que abarcam a auditoria nos serviços de saúde.

\section{MÉTODOS}

Optou-se pela revisão sistemática de caráter exploratório, com abordagem qualitativa. Esse tipo de pesquisa objetiva desvendar, recolher e analisar principais contribuições teóricas sobre um determinado fato, recurso importante da prática baseada em evidências em saúde, que consiste em uma forma de síntese dos resultados de pesquisas relacionados com um problema específico (GALVÃO, et al., 2004).

Realizou-se a busca de estudos em periódicos nacionais publicados entre os anos 2014 ao primeiro bimestre de 2018 , na base de dados da Literatura Latino-Americana em Ciências de Saúde (LILACS), e na Scientific Electronic Library Online (SciELO). Todavia, dados sobre o assunto são escassos, nas dimensões propostas deste estudo, utilizou-se o buscador Google acadêmico para acessar publicações. Para tanto, utilizaram-se os descritores: Auditoria, Qualidade da assistência, Planos de saúde, Registros de enfermagem, Ferramenta de gestão. Conforme Descritores em Ciências da Saúde (DECS, 2015).

Os artigos receberam leitura exploratória e analítica, com a finalidade de identificar a validade do documento para o presente trabalho; em seguida, resumido as informações sobre o objeto de estudo para posteriormente, receberem uma leitura de característica interpretativa, com o problema proposto, foram considerados aspectos qualitativos, focando a importância do processo de auditoria para discussões sobre a temática. 


\section{RESULTADOS E DISCUSSÃO}

\subsection{Auditoria: viés histórico e contemporâneo}

Conceitualmente o significado da palavra auditor, na língua português, deriva da nomenclatura inglesa ' audit" qual denota a ação de examinar, acertar, corrigir, certificar, em convergência ao âmbito da área da saúde, estigmatizada, como um método usado para avaliação e domínio das ações que refletem na qualidade da assistência prestada ao paciente/cliente (CLAUDINO, et al. 2013; SIQUEIRA, 2014; BOTELHO e FARIA, 2016; FILHO, et al. 2016; SILVA, et al. 2017; SCHEK, et al. 2018).

A prestação de serviços na saúde existe muitos séculos e a auditoria surge na área da saúde pela primeira vez no estudo realizado pelo médico George Gray Ward, nos Estados Unidos, em 1918, no qual foi verificada a qualidade da assistência médica prestada ao paciente por meio dos registros em prontuário (SANTOS, et al. 2013; SIQUEIRA, 2014; SILVA, 2016; SEIFFERT, 2016; DORNE e HUNGARE, 2017; GARCIA, et al 2018).

Deste modo há diferentes conceituações para auditoria, comumente é caracterizada por avaliação sistemática e formal de uma atividade, por alguém não envolvido diretamente na execução, este, deve observar a exatidão, a integridade e a autenticidade de tais demonstrações, comumente em registros e documentos, uma espécie de sistema de revisão de controle qual tem como objetivo informar a administração sobre a eficiência e a eficácia dos programas em desenvolvimento (SILVA e SANTO, 2013; REIS e KITAMURA, 2016; CARMO, 2017; BORTOLOSSO, et al. 2017).

No que tange a âmbito nacional a Norma Regulamentadora Brasileira ISO 9000:2000, classifica a auditoria como uma atividade de coleta de dados para verificar o atendimento aos requisitos especificados, buscando evidências de concordâncias, aferindo as necessidades de ações corretivas ou de aperfeiçoamento, não devendo identificar os culpados pelo não desacordo e sim, propor soluções para que sejam suprimidas (CECCON, et al. 2013; SIQUEIRA, 2014; BRANDÃO e SILVA, 2015; OLIVEIRA, et al. 2017).

Portanto, auditoria é um instrumento de administração utilizado na avaliação da qualidade do cuidado, baseado na checagem entre a assistência prestada e os padrões de assistência considerados aceitáveis (SILVA e SANTO, 2013; VIANA, et al. 2016; COSTA, 2017; LOURENÇO e MALHEIROS, 2017).

\subsection{Compreendendo a auditoria a âmbito nacional}

O desenvolvimento da instrumentalização da auditoria a âmbito nacional, é denotada, explorada e adaptada as nossas realidades apenas a meio século atrás, tendo seus primeiros trabalhos desenvolvidos operacionalmente na área de saúde na década de 60 (CLAUDINO, et al. 2013; SIQUEIRA, 2014; BOTELHO e FARIA, 2016; FILHO, et al. 2016; SILVA, et al. 2017; SCHEK, et al. 2018).

Na esfera das ações público da saúde, antes de 1976 as atividades eram realizadas pelos 
supervisores do Instituto Nacional de Previdência Social (INPS) não havendo auditorias diretas em hospitais, em 1983 o cargo de médico-auditor foi reconhecido e a auditoria iniciou-se nos próprios hospitais (SILVA e SANTO, 2013; REIS e KITAMURA， 2016; CARMO， 2017; BORTOLOSSO, et al. 2017).

No Sistema Único de Saúde (SUS), em 1990, quando criou-se um sistema que regulamentaria as regras de auditoria, e somente foi instituído em 1993, pela Lei n. ${ }^{\circ} 8.689$, com o objetivo de nortear e controlar os prestadores de serviços de saúde, qual foi de extrema necessidade a época (SANTOS, et al. 2013; SIQUEIRA, 2014; SILVA, 2016; SEIFFERT, 2016; DORNE e HUNGARE, 2017; SOUSA, et al. 2018).

A Lei n. ${ }^{\circ} 8.080$, de 1990, ao prever a criação do Sistema Nacional de Auditoria (SNA), constituiu as instâncias de gestão do SUS quais suas primazias eram de seguir, controlar e avaliar as ações e serviços de saúde a âmbito nacional, ficando reservada à União a competência privativa de estabelecer o SNA, e coordenar a avaliação técnica e financeira do SUS em todo o território nacional em colaboração técnica com os governos (CECCON, et al. 2013; SIQUEIRA, 2014; BRANDÃO e SILVA, 2015; OLIVEIRA, et al. 2017).

Neste contexto, por tratar designadamente da área da saúde, o SNA se constitui num sistema atípico, singular, diferenciado, complementar aos sistemas de controle interno e externo legítimo qual sua fundamentação é notada como reguladora da temática (SILVA e SANTO, 2013; VIANA, et al. 2016; COSTA, 2017; LOURENÇO e MALHEIROS, 2017).
Para tal, criada pelo Ministério da Saúde (MS), a Agência Nacional de Saúde Suplementar nasceu pela Lei $\mathrm{n}^{\circ}$ 9.961, de 28 de janeiro de 2000, como instância reguladora e responsável pelo setor de planos de saúde no Brasil (CLAUDINO, et al. 2013; SIQUEIRA, 2014; BOTELHO e FARIA, 2016; FILHO, et al. 2016; SILVA, et al. 2017; GARCIA, et al. 2018).

Contudo, em analogia ao setor privado, da Agência Nacional de Saúde Suplementar (ANS), foi na década de 50 quais instituições hospitalares privadas consolidaram-se prestadoras de serviço atendendo a classe média emergente, e com isso a saúde suplementar passou a conviver com o sistema público. Atualmente o setor brasileiro de planos e seguros de saúde é o segundo maior sistema privado de saúde do mundo (SILVA e SANTO, 2013; VIANA, et al. 2016; COSTA, 2017; LOURENÇO e MALHEIROS, 2017).

Contextualizando, os planos de saúde tiveram sua origem na fundação das Santas Casas de Misericórdia, instituições vinculadas à Igreja Católica com ações caritativas e filantrópicas, quais emanciparam ideários de qualidade e eficácias a população (SANTOS, et al. 2013; SIQUEIRA, 2014; SILVA, 2016; SEIFFERT, 2016; DORNE e HUNGARE, 2017; SOUSA, et al .2018).

\subsection{A dinâmica do processo de classificação da auditoria}

A classificação da auditoria está sujeita a variação de sua execução; (analítica ou operativa), o método (prospectiva, concorrente ou retrospectiva), a forma de intervenção (interna ou 
externa), o tempo (contínua ou periódica), a natureza (regular ou especial); e ao limite (total ou parcial) (SILVA e SANTO, 2013; REIS e KITAMURA, 2016; CARMO, 2017; BORTOLOSSO, et al. 2017).

Neste contexto, a auditoria analítica consiste em sua primazia em análise de relatórios, processos e documentos, com a finalidade de subsidiar a verificação in loco, dos referidos processos metodológicos em saúde, já a auditoria operativa consiste na verificação in loco do documentos e situações, para determinar a adequação, a conformidade, economicidade, legalidade, legitimidade, eficiência, eficácia (SANTOS, et al. 2013; SIQUEIRA, 2014; SILVA, 2016; SEIFFERT, 2016; DORNE e HUNGARE, 2017; SOUSA, et al. 2018).

Quanto ao processo do método, a auditoria em saúde pode ser classificada em prospectiva, concorrente ou retrospectiva, a auditoria prospectiva refere-se a avaliação dos procedimentos médicos hospitalares antes de sua realização é utilizado no sistema de saúde suplementar, exemplificando, comumente na emissão de um parecer, designado pela operadora de plano de saúde sobre algum tratamento ou procedimento (CLAUDINO, et al. 2013; SIQUEIRA, 2014; BOTELHO e FARIA, 2016; FILHO, et al. 2016; SILVA, et al. 2017; SCHEK, et al. 2018).

Entretanto, a ação de auditar pode ser classificada em: interna e externa, pois a auditoria Interna é realizada por elementos da própria instituição hospitalar, devidamente informados e treinados, e a auditoria externa ou independente é realizada por profissionais que não pertencem à instituição (SILVA e SANTO, 2013; VIANA, et al. 2016; COSTA, 2017; LOURENÇO e MALHEIROS, 2017).

Deste modo pode ser classificada em contínua ou periódica, pois a dicotomia entre auditoria contínua é que a mesma é realizada em períodos determinados, sem sofrer interrupções, e a auditoria periódica relaciona-se a certos períodos, não possuindo características de continuidade (CLAUDINO, et al. 2013; SIQUEIRA, 2014; BOTELHO e FARIA, 2016; FILHO, et al. 2016; SILVA, et al. 2017; SCHEK, et al. 2018).

Especifica-se ainda de acordo com o limite, esta pode ser classificada em total ou parcial, deste modo a auditoria total abrange todos os setores da instituição, todos os documentos referentes a prestação de serviço e regulamentares já a auditoria parcial abrange apenas a alguns serviços da instituição (SANTOS, et al. 2013; SIQUEIRA, 2014; SILVA, 2016; SEIFFERT, 2016; DORNE e HUNGARE, 2017; SOUSA, et al. 2018).

\subsection{Auditoria e gestão: um dilema organizacional}

O processo inerente a auditoria é caracterizada como ferramentas administrativas, quais são usadas para identificação e analise de problemas, quais são resultado indesejado ou um desafio a ser corrigido (CECCON, et al. 2013; SIQUEIRA, 2014; BRANDÃO e SILVA, 2015; OLIVEIRA, et al. 2017).

Contudo, a dinâmica das organizações no mercado corroborando com a ausência de conhecimento desses administradores, qual 
fomenta-se a necessidade/auxílio da auditoria como ferramenta administrativa para obtenção de informações, atividade financeira, redução de custos e qualitativos à gestão hospitalar (SILVA e SANTO, 2013; VIANA, et al. 2016; COSTA, 2017; LOURENÇO e MALHEIROS, 2017).

Para tal, a dinâmica de auditar é capaz de eliminar desperdícios, simplifica tarefas evitando inúmeros riscos, frequentes erros, a auditoria tem de estar aliada ao planejamento hospitalar, deste modo a auditoria torna-se fundamental para uma boa gestão (CLAUDINO, et al. 2013; SIQUEIRA, 2014; BOTELHO e FARIA, 2016; FILHO, et al. 2016; SILVA, et al. 2017; SCHEK, et al. 2018).

Neste contexto a auditoria hospitalar desempenha papel fundamental para as instituições hospitalares, em nível financeirocomercial, avaliando consumos e cobranças realizadas pela instituição $(\mathrm{CECCON}$, et al. 2013; SIQUEIRA, 2014; BRANDÃO e SILVA, 2015; OLIVEIRA, et al. 2017).

Em linha gerais, a avaliação da auditoria no contexto hospitalar faz-se em base a análise do prontuário do paciente no qual é relatado quando devidamente escrito, horários, datas e parecer técnico dos profissionais que assistem o paciente, portanto, o profissional auditor em qualquer área de atuação contribui para a empresa pública ou privada, no sentido de promover e manter a saúde do usuário (SILVA e SANTO, 2013; REIS e KITAMURA， 2016; CARMO， 2017; BORTOLOSSO, et al. 2017).

A dinamização da auditoria pode atuar como a nível estrutural com órgão de assessoria apoiando-se no modelo de gestão adotado pelo órgão de gestão da organização, deste modo culminando em redução dos riscos (CLAUDINO, et al. 2013; SIQUEIRA, 2014; BOTELHO e FARIA, 2016; FILHO, et al. 2016; SILVA, et al. 2017; GARCIA, et al. 2018).

Deste modo, auditoria eficiente, depende do auditor busca conhecer detalhes mínimos sobre as aspirações filosóficas da instituição, ter a vivencia em contratos de atendimento aos clientes compreender, prestação de serviços, comportamento ético e sigiloso, analisando documentos e preparar parecer técnico (SANTOS, et al. 2013; SIQUEIRA, 2014; SILVA，2016; SEIFFERT，2016; DORNE e HUNGARE, 2017; SOUSA, et al. 2018).

Após a auditoria os dados e informações podem ser usados para criar medidas ou indicadores para monitorar e melhorar seu desempenho, produtividade; custo; prazo; satisfação do cliente; participação no mercado; desperdício e qualidade total (SILVA e SANTO, 2013; VIANA, et al. 2016; COSTA, 2017; LOURENÇO e MALHEIROS, 2017).

A auditoria como ferramenta de gestão tem valor fundamental para as empresas hospitalares de acordo com as informações levantadas na operacionalização, porém se às organizações não houver liderança e compromisso com a qualidade, e os administradores não garantirem às equipes o tempo e os recursos necessários, se a cultura organizacional não for mudada, e se as pessoas não forem fortalecidas pela educação, esta ferramenta fundamental não cumprira com seu proposito situacional (CECCON, et al. 2013; SIQUEIRA, 2014; 
BRANDÃO e SILVA, 2015; OLIVEIRA, et al. 2017).

Portanto, a auditoria em saúde em conjunto com a gestão hospitalar para terem êxito exigem um comprometimento de todo efetivo envolvido, pois, não tem por finalidade avaliar o desempenho de um indivíduo ou grupo, mas de melhorar a assistência ao paciente (SILVA e SANTO, 2013; REIS e KITAMURA, 2016; CARMO, 2017; BORTOLOSSO, et al. 2017).

\section{CONCLUSÃO}

Aspectos de interconexões acerca da explicações e extensões do instrumento de auditoria no campo da saúde na contemporaneidade foram obtidos, e suas implicações, por meio desta averiguação proporcionam proeminência social, profissional e acadêmica. Ao que tange a pratica do processo de auditar conclui-se que o mesmo apresenta grande valia no que tange ao progresso da aparelhamento hospitalar em desenvolvimento de novas metodologia influenciando diretamente nos custos e qualidade ao usuário da saúde.

Para tal, assim como as auditorias de contas médicas e de enfermagem, é necessário ter capacidade, ao auditor, habilidades técnicas e preparo intelectual, que é a abertura para o crescimento de uma organização e o mesmo ter controle de exercer uma auditoria imparcial e comprovar dados prestados seja de extrema confiabilidade. Neste contexto, o método de auditoria é muito importante, com função de fiscalizar, analisar e relatar dados e alternativas de atos para os hospitais, este agiliza os frutos adquiridos. A auditoria como instrumento para a excelência da gestão hospitalar, a mesma não pretende em sua primazia apenas identificar anormalidades, e sim ajustar. Portanto, a agilidade de auditoria hospitalar é destacada como ferramenta de gestão e inspeção apropriado às necessidades de gerenciamento, modelo de mensuração de informações chegando a resultados satisfatórios.

As reivindicações contemporâneas da saúde na qualidade do serviço e redução de custos, faz com que o profissional seja qualificado no manejo da auditoria seja componente da gestão e administração no sistema público e privado tendo o mesmo objetivo final uma melhor prestação de serviço ao usuário.

$\mathrm{Na}$ área da enfermagem, o enfermeiro auditor tem grande importância quando a finalidade é assegurar que todos os procedimentos sejam realizados de forma adequada, trazendo como objetivo da auditoria os benefícios para a instituição e para o paciente.

Diante desta situação, observa a falta de valorização da importância dos registros e anotações de forma coerente e completa, e proposta de melhoria, através do preambulo de alguns autores, observou a necessidade de oferecer treinamentos e educação continuada, para o planejamento do trato com a auditoria.

Por fim, mesmo com relatos do crescimento do alento a problemática percebeu-se que há escassez da literatura sobre a luz da temática desta forma, evidencia-se a necessidade da realização de novos estudos a fim de investigar e analisar, os processos de auditoria. 


\section{REFERÊNCIAS}

ALELUIA, Ítalo Ricardo Santos; SANTOS, Fabiane Costa. Auditoria em fisioterapia no Sistema Único de Saúde: proposta de um protocolo específico. Fisioterapia em Movimento, v. 26, n. 4, 2013.

AMARAL FILHO, Roberto Cesar de Almeida et al. Importância do registro de enfermagem para o faturamento hospitalar: revisão da literatura. Revista de trabalhos acadêmicos universo-são gonçalo, n. 1, p. 247-263, 2016.

BORTOLOSSO, Heleia et al. Métodos de auditoria de sistemas de gestão de segurança e saúde no trabalho: uma revisão sistemática da literatura. Ingeniería industrial. Actualidad y Nuevas Tendencias, n. 18, p. 45-58, 2017.

BOTELHO, Marcos Ribeiro; DE FARIA, Mário Parreiras. Auditoria em segurança e saúde no trabalho em empresas produtoras de ferro-gusa no estado de minas gerais, brasil. Revista baiana da inspeção do trabalho, p. 75, 2016.

BRANDÃO, Ana Claudia Soares; SILVA, JRA. A contribuição dos sistemas de informação em saúde (SIS) para o processo de auditoria do sus. Revista Atualiza Saúde, v. 17, 2015.

CAMELO, Silvia Helena Henriques et al. Auditoria de enfermagem e a qualidade da assistência à saúde: uma revisão da literatura.

Revista Eletrônica de Enfermagem, v. 11, n. 4, p. 1018-25, 2009.

CARMO, Tatiane Garcia do. Impacto financeiro dos serviços de auditoria do SUS no programa Farmácia Popular. Revista Saúde e Desenvolvimento, v. 11, n. 7, p. 131-145, 2017.

CECCON, Roger Flores et al. Enfermagem, auditoria e regulação em saúde: um relato de experiência. Revista Mineira de Enfermagem, v. 17, n. 3, p. 695-704, 2013.

CLAUDINO, Hellen Gomes et al. Auditoria em registros de enfermagem: revisão integrativa da literatura. Rev. enferm. UERJ, v. 21, n. 3, p. 397 402, 2013.

COSTA, Djalma Ribeiro. Auditoria de contas ambulatoriais no sistema único de saúde no estado do Piauí. Revista de Administração em Saúde, v. 17, n. 68, 2017.

DA SILVA, César Carriço. A auditoria e a sua perspectiva na área da saúde. Signorelli digital revista de produção científica, p. 182, 2016.

DA SILVAI, Maria Verônica Sales et al. Limites e possibilidades da auditoria em enfermagem e seus aspectos teóricos e práticos. Revista Brasileira de Enfermagem, v. 65, n. 3, p. 535538, 2012.

DE LIMA PASSOS, Maria Leonice et al. Auditoria de enfermagem: conhecimento de profissionais em hospital público de referência. Revista da Rede de Enfermagem do Nordeste, v. 13, n. 5, p. 1025-1033, 2012.

D'INNOCENZO, Maria et al. Indicadores, auditorias, certificações: ferramentas de qualidade para gestão em saúde. In: Indicadores, auditorias, certificações: ferramentas de qualidade para gestão em saúde. 2010. p. 208-208.

DORNE, Juliane; HUNGARE, Jaqueline Volpato. Conhecimentos teóricos de auditoria em enfermagem. Revista UNINGA Review, v. 15, $\mathrm{n}$. 1, p. 8-8, 2017.

GALVÃO, C. M.; SAWADA, N. O.; TREVIZAN, M. A. Revisão sistemática.Rev Latino-am enfermagem, v. 12, n. 3, p. 549-56, 2004. Janeiro, mar. 2012.

GARCIA, Thayse Tobar; DA SILVA FERREIRA, Wellington Fernando; DA SILVA, Alice. PROCESSO DE AUDITORIA EM ENFERMAGEM E SUAS DIMENSÕES NA ASSISTÊNCIA AO PACIENTE: UMA REVISÃO SISTEMÁTICA DE LITERATURA. Ciência \& Desenvolvimento-Revista Eletrônica da FAINOR, v. 11, n. 1, 2018.

LOURENÇO, Abner Eliezer; MALHEIROS, Izabel. Avaliação da qualidade da assistência de enfermagem através de auditoria: uma revisão bibliográfica. Miríade Científica, v. 1, n. 2, 2017.

MOIMAZ, Suzely Adas Saliba et al. Auditoria na Saúde: justificativas de glosas no setor odontológico. J Health Sci Inst, v. 30, n. 2, p. 112-6, 2012.

OLIVEIRA, Edilaine Cristina de et al. Cuidados pós-alta em pacientes idosos com sequelas de 
acidente vascular cerebral: planejamento de alta hospitalar. Revista Saúde e Desenvolvimento, v. 11, n. 9, p. 172-197, 2017.

REIS, Felipe Rovere Diniz; KITAMURA, Satoshi. O controle estatal em saúde e segurança no trabalho e a auditoria do Programa de Controle Médico de Saúde Ocupacional. Rev Bras Med Trab, v. 14, n. 1, p. 52-9, 2016.

ROSA, Liliane de Abreu et al. Auditoria como estratégia de avaliação dos registros de enfermagem em unidade de internação pediátrica. Revista Mineira de Enfermagem, v. 16, n. 4, p. 553-546, 2012.

SANTOS, Cristina Almeida dos et al. A auditoria e o enfermeiro como ferramentas de aperfeiçoamento do SUS. Revista Baiana de Saúde Pública, v. 36, n. 2, p. 539, 2013.

SCARPARO, Ariane Fazzolo et al. Auditoria em Enfermagem: identificando sua concepção e métodos. Revista Brasileira de Enfermagem, v. 61, n. 3, p. 302-305, 2008.

SCHEK, Gabriele et al. A produção do conhecimento acerca da auditoria de enfermagem: aspectos teóricos e práticos. Revista de Enfermagem, v. 13, n. 13, p. 78-94, 2018.

SEIFFERT, Mari Elizabete Bernardini. Auditoria de Sistemas de Gestão-Princípios, Procedimentos e Práticas com Ênfase nas Normas ISO (9001, 14001, 22000) e OHSAS 18001. Revista Brasileira de Contabilidade, n. 219, p. 90-90, 2016.

SILVA, Agneta Torres da; SANTO, Eniel do Espírito. A Auditoria como Ferramenta para a Excelência da Gestão Hospitalar. Revista saúde e desenvolvimento, v. 3, n. 2, p. 43-60, 2013.

SILVA, Carla Aparecida et al. Auditoria como instrumento de gestão na Atenção Primária à Saúde. Gerais: Revista de Saúde Pública do SUS/MG, v. 3, n. 1, p. 72-80, 2017.

SIQUEIRA, Patrícia Lopes de Freitas. Auditoria em saúde e atribuições do enfermeiro auditor.

Caderno saúde e desenvolvimento, v. 4, n. 3, p. 519, 2014.
SOUSA, Maria das Graças de Melo et al. A sistematização da assistência de enfermagem no contexto da auditoria hospitalar. Revista uningá review, v. 25, n. 3, 2018.

VIANA, Carla Denise et al. Implantação da auditoria concorrente de enfermagem: um relato de experiência. Texto \& contexto enfermagem. Florianópolis. Vol. 25, n. 1 (2016), p. e3250014, 2016.

\section{Elia Machado de Oliveira}

Enfermeira, Mestre em Cirurgia; Especialista em Assistência de Enfermagem ao Paciente em Estado Crítico, Especialista em Comunicação Organizacional, Especialista em Auditoria de Serviços de Saúde.

\begin{tabular}{l}
\hline Wellington Fernando da Silva Ferreira \\
Enfermeiro, Especialista em Saúde do Idoso e \\
Gerontologia.
\end{tabular}

